\title{
ISOSPECTRAL FINITENESS ON CONVEX COCOMPACT HYPERBOLIC 3-MANIFOLDS
}

\author{
GILLES COURTOIS AND INKANG KIM
}

\begin{abstract}
In this paper we show that a given set of lengths of closed geodesics, there are only finitely many convex cocompact hyperbolic 3-manifolds with that specified length spectrum, homotopy equivalent to a given 3-manifold without a handlebody factor, up to orientation preserving isometries.
\end{abstract}

\section{INTRODUCTION}

One of the main theme of Riemannian geometry is to find a criterion for two manifolds to be isometric. Along the development in this direction, the study of closed geodesics proved to be very useful in many cases. The length spectrum $\Lambda(M)$ of a Riemannian manifold $M$ is the set of lengths of closed geodesics with multiplicity. If we consider the length spectrum without multiplicity, we will say so explicitly. There exists a famous conjecture called conjugacy problem. If two compact negatively curved manifolds have the same marked length spectrum, then it is conjectured that they are isometric. There is very little progress on this conjecture except when one manifold is locally symmetric [3] or both are surfaces [38, 20].

There is an anologous conjecture. It states that the set of negatively curved metrics on a fixed compact manifold with a fixed set of lengths of closed geodesics, forms a compact (or finite) set in the space of Riemannian metrics. One can rephrase this as an unmarked length rigidity. There is also very little progress in this direction. There is a close relationship between length spectrum and Laplace spectrum. For negatively curved compact manifolds, the latter determines the former, see

\footnotetext{
${ }^{1} 2000$ Mathematics Subject Classification. 51M10, 57S25.

${ }^{2}$ Key words and phrases. Isospectral rigidity, convex cocompact hyperbolic manifold, injectivity radius, algebraic convergence, incompressible core.

${ }^{3}$ The second author gratefully acknowledges the partial support of grant (NRF-2014R1A2A2A01005574), CNRS, Ecole Polytechnique and l'Institut de Mathématiques de Jussieu during his visit.
} 
[48]. For surface case, two notions are equivalent by Selberg trace formula. McKean in the 70's showed [34] that there exists only finite number of hyperbolic metrics on a surface with a given Laplace spectrum. Later Osgood, Phillips and Sarnak [37] showed the compactness of Laplacian isospectral metrics on a closed surface. Much later, Brooks, Perry and Petersen [7] showed the same result for closed 3-manifolds near a metric of constant curvature. More recently, Croke and Sharafutdinov [20] showed the non-existence of smooth one-parameter Laplacian isospectral deformation of a closed negatively curved manifold. For Laplace spectrum and related problems, see [21, 22, 39, 44, 49.

In this paper, we deal with infinite volume case and prove a finiteness result for convex cocompact real hyperbolic 3-manifold. The main theorem is:

Theorem 1. Let $M$ be a convex cocompact real hyperbolic 3-manifold (which is not a solid torus) with a length spectrum $\Lambda$ with multiplicity. Suppose $\pi_{1}(M)$ does not have any free factor, i.e., $\pi_{1}(M)$ cannot be represented as $G * F_{n}$ where $F_{n}, n \geq 1$ is a free group of $n$-generators. Then there exist only finite number of convex cocompact hyperbolic 3manifolds homotopy equivalent to $M$ with the length spectrum $\Lambda$.

For convex cocompact boundary-incompressible hyperbolic 3-manifold case, it was treated in [29] but the proof was incomplete. In this paper we give a complete proof for boundary incompressible case in Theorem 6 and a generalization to boundary compressible case in our main theorem.

For reader's convenience, we outline the proof of the main theorem. Fix a convex cocompact hyperbolic 3-manifold $M$. We argue by contradiction considering a sequence of hyperbolic 3-manifolds homotopy equivalent to $M$ with a fixed length spectrum. The basic idea is to find an algebraically convergent subsequence to derive a contradiction. This process reduces the unmarked length spectrum problem to a marked length spectrum one. In Theorem 6, we show that given a discrete set $\Lambda$, there are only finitely many convex cocompact hyperbolic 3-manifolds homotopy equivalent to $M$ with incompressible boundary, whose length spectrum without multiplicity is contained in $\Lambda$. This follows from Theorem 5 that one can find an algebraically convergent subsequence if there is a lower bound for the injectivity radius in boundary incompressible case.

For general case in the sense that the boundary might be compressible, we decompose the manifold $M$ into the union of incompressible core $\cup M_{j}$ connected by 1 -handles where each $M_{j}$ has incompressible 
boundary. If $N_{i}$ is an infinite sequence of convex cocompact hyperbolic 3-manifolds homotopy equivalent to $M$ and with a fixed length spectrum with multiplicity, using Theorem 6, we can assume that the covering manifolds $N_{i}^{j}$ corresponding to $M_{j}$ are all isometric to each other for a fixed $j$. Next if the lengths of the 1-handles attached are all bounded, then by Theorem 8, it is finished. If the length of some handle goes to infinity, we argue that $N_{i}$ do not have the same length spectrum with multiplicity. The general case is treated in Thereom 9 .

In section 7, we treat the similar result for the convex cocompact surface group representations in rank one semisimple Lie groups and obtain the following.

Theorem 2. Let $G$ be a semisimple real Lie group of rank one of noncompact type. Fix $\Lambda$ a discrete set of positive real numbers, and a closed surface $S$ of genus $\geq 2$. Then the set of convex cocompact representations $\rho: \pi_{1}(S) \rightarrow G$ with $\Lambda_{\rho}=\Lambda$ is finite up to conjugacy and the change of marking. Here $\Lambda_{\rho}$ is the set of translation lengths of $\rho\left(\pi_{1}(S)\right)$.

We hope that we can generalize the argument to non-surface group case in any semisimple Lie groups in the near future.

Acknowledgements. The authors are grateful to R. Canary for pointing out the reference [15] and to anonymous referees for pointing out some inaccuracies in the earlier version, specially Chris Croke for having pointed out a mistake in a previous version of this paper.

\section{PRELIMINARIES}

A real hyperbolic manifold is a locally symmetric Riemannian manifold with constant negative sectional curvature, which is of the form $H_{\mathbb{R}}^{n} / \Gamma$ where $\Gamma$ is a torsion free discrete subgroup of $I s o\left(H_{\mathbb{R}}^{n}\right)$, the isometry group of $H_{\mathbb{R}}^{n}$. $H_{\mathbb{R}}^{n}$ is topologically an open unit ball in $\mathbb{R}^{n}$ and one can compactify it by attaching $S^{n-1}$ which is called the sphere at infinity. Iso $\left(H_{\mathbb{R}}^{n}\right)$ naturally acts on $S^{n-1}$ as conformal maps. A limit set $L_{\Gamma}$ of $\Gamma$ is $S^{n-1} \cap \overline{\Gamma x_{0}}$ where $x_{0} \in H_{\mathbb{R}}^{n}$ is a base point and the closure is with respect to the Euclidean topology in the unit ball. $\Omega(\Gamma)=S^{n-1} \backslash L_{\Gamma}$ is called the domain of discontinuity which is the largest $\Gamma$-invariant open set of $S^{n-1}$ on which $\Gamma$ acts properly discontinuously. For $n=3$, $S^{2}=\hat{\mathbb{C}}$ and if $\Gamma$ is not abelian, $\Omega(\Gamma)$ inherits a hyperbolic metric. In this case, $\Omega(\Gamma) / \Gamma$ is called the conformal boundary of $H_{\mathbb{R}}^{3} / \Gamma$ which is a hyperbolic surface.

A convex hull $C H(\Gamma)$ of $\Gamma$ is the smallest closed convex set in $H_{\mathbb{R}}^{n}$ which is invariant under $\Gamma . C H(\Gamma) / \Gamma$ is called the convex core $C(\Gamma)$ of 
$H_{\mathbb{R}}^{n} / \Gamma$. If $C(\Gamma)$ is compact, $\Gamma$ is called convex cocompact. For $n=3$, the boundary of the convex core inherits a hyperbolic metric with respect to the path metric on it. For any point $x \in H_{\mathbb{R}}^{n}$, there is a closest point in $C H(\Gamma)$ since $C H(\Gamma)$ is a closed convex set, so there exists a natural map

$$
r: \Omega(\Gamma) / \Gamma \rightarrow \partial C(\Gamma),
$$

called the nearest point retraction from the conformal boundary to the convex core boundary. Using this map, one can easily see that $C(\Gamma)$ and $\left(H_{\mathbb{R}}^{n} \cup \Omega(\Gamma)\right) / \Gamma$ are homeomorphic if $\Gamma$ is convex cocompact and Zariski dense. In fact, $C(\Gamma)$ is a deformation retract of $H_{\mathbb{R}}^{n} / \Gamma$. This is true for general negatively curved manifolds. If $\partial\left(H_{\mathbb{R}}^{3} / \Gamma \cup \Omega(\Gamma) / \Gamma\right)$ is incompressible, this retraction map is $K$-Lipschitz for some universal $K$ independent of the manifold [40]. But if the manifold has compressible boundary, such a universal constant does not exist.

Definition 1. Let $M$ be a Riemannian manifold without boundary. For $x$, the injectivity radius inj $(x)$ of $x$ is defined to be the supremum of $r>0$ such that the metric ball $B_{r}(x)$ in $M$ is isometric to the $r$-ball in the universal cover $\tilde{M}$. For a compact convex subset $A \subset M$, the injectivity radius of $A$ is defined to be the maximum of $r>0$ such that a finite number of $r$-balls $\bar{B}_{r}\left(x_{i}\right)$ (homeomorphic to the closed $r$-ball in the universal cover) contained in $A$ whose union, in such a way that $d\left(x_{i}, x_{j}\right)>r, \cup \bar{B}_{r}$ is homotopy equivalent to $A$, and together with a finite number of $r$-balls $B_{r}\left(y_{j}\right)$, not necessarily contained in $A$, in such a way that $d\left(y_{i}, y_{j}\right)>r, d\left(x_{i}, y_{j}\right)>r$, can cover $A$.

The injectivity radius of the subset of the manifold is defined in the way that if it has a long thin compression 1-handle, then the injectivity radius is small. We will use this definition to deal with the 3-manifold with compressible boundary, specially the convex core of such a 3manifold. See section 5 .

Definition 2. Let $\left(M_{i}, \omega_{i}\right)$ be a sequence of hyperbolic manifolds with orthonormal base frames. Then $\left(M_{i}, \omega_{i}\right)$ converge geometrically to $(M, \omega)$ if and only if, for each compact submanifold $K \subset M$ containing the base frame $\omega$, there are smooth embeddings $f_{i}: K \rightarrow M_{i}$, defined for all sufficiently large $i$, such that $f_{i}$ sends $\omega$ to $\omega_{i}$ and $f_{i}$ tends to an isometry in $C^{\infty}$ topology, i.e., all the derivatives of any order converge uniformly on $K$ to the derivatives of the same order of an isometry. See [2].

In practice, we use $\left(M_{i}, x_{i}\right)$ where $x_{i}$ is a base point of the frame $w_{i}$ since the set of base frames at $x_{i}$ is compact. This is a sequence of pointed manifolds instead of a sequence of manifolds with base frames. 
It is known that such $M$ exists if the injectivity radius at the base point of the base frame is bounded below for all $i$. See [35, 2]. For general Riemannian metrics, Gromov's compactness theorem states that: the set of isometry classes of closed Riemannian $n$-manifolds with uniformly bounded curvatures, diameters bounded above, and volumes bounded below, is precompact in the $C^{1, \alpha}$ topology for any $\alpha<1$. More precisely, for any sequence of Riemannian $n$-manifolds $\left(M_{i}, g_{i}\right)$ satisfying the above conditions, there exist a subsequence, also called $\left(M_{i}, g_{i}\right)$, and diffeomorphisms $\phi_{i}: M_{\infty} \rightarrow M_{i}$ so that the metrics $\phi_{i}^{*} g_{i}$ converge in the $C^{1, \alpha}$ topology to a limit metric $g_{\infty}$ on $M_{\infty}$.

A compact irreducible 3-manifold $M$ has an incompressible core, which is a collection $\left\{M_{1}, \cdots, M_{n}\right\}$ of submanifolds of $M$ such that $M$ is obtained from this collection by adding 1-handles and each $M_{i}$ has incomressible boundary, see [33. In this paper, we deal with 3-manifolds with almost incompressible boundary in the sense of McCullough [32, i.e., $\pi_{1}(M)$ does not have any free group factor.

A simple closed curve in a boundary $\partial M$ of $M$ is called a meridian if it is nontrivial in $\partial M$ but trivial in $M$. By loop theorem, it bounds a compression disk. The following lemma is practical. Before we prove the lemma, we need some terminologies.

Any topological annulus is conformally equivalent to a Euclidean annulus, i.e., an annulus bounded by two concentric circles. The modulus of an annulus $A$ bouned by concentric circles of radius $r_{2}>r_{1}$ is

$$
\bmod (A)=\frac{1}{2 \pi} \log \left(\frac{r_{2}}{r_{1}}\right) .
$$

In 43] (Theorem 5.2), it is shown that if a conformal boundary contains a meridian of length $L \leq 1$, then it contains a topological annulus in the universal cover with modulus $C / L$ where $C$ can be taken as $\frac{\pi}{\sqrt{e}}$.

Lemma 1. For a given $\epsilon$ there is $K(\epsilon)$ such that $K(\epsilon) \rightarrow \infty$ as $\epsilon \rightarrow 0$ with the following conditions. Let $M$ be a hyperbolic 3-manifold with a compressible boundary. Let $m$ be a meridian which bounds a compression disk $D$. If the length of the meridian in a conformal boundary is $\epsilon$, then the length of the geodesic intersecting $D$ transversely is bigger than $K(\epsilon)$.

Proof: Suppose $\epsilon$ is small so that the modulus of a topological annulus $R$ whose core is a meridian $m$, is $C / \epsilon>0.6$. Lift it to the universal cover of $M$. Since $m$ is a meridan, $R$ lifts to again an annulus $\tilde{R}$ in $\hat{\mathbb{C}}$. Then by [24], there is a Euclidean annulus $A$ inside $\tilde{R}$ with modulus

$$
\bmod (A) \geq \bmod (\tilde{R})-\frac{1}{\pi} \log 2(1+\sqrt{2}) \geq \bmod (\tilde{R})-0.502 .
$$


Let $C H(A)$ be the convex hull of the annulus $A$. If a geodesic $\gamma$ intersects the compression disk $D$ transversely, $\tilde{\gamma}$ should pass through $C H(A)$, and so its length is at least $2 \pi \bmod (A)$. If $\epsilon$ tends to zero, $\bmod (\tilde{R})$ tends to infinity, so does $2 \pi \bmod (A)$.

Once there is a lower bound for the lengths of meridians, one can compare the lengths of curves in conformal and convex core boundary. See [5]. From now on we drop the subscript $\mathbb{R}$ for simplicity to denote the real hyperbolic 3 -space $H^{3}$.

Theorem 3. For any $\epsilon>0$, there is a constant $K>0$ depending only on $\epsilon$ with the following conditions. Let $\Gamma$ be a finitely generated Kleinian group without torsion such that the shortest meridian length is greater than $\epsilon$. Let $C(\Gamma)$ be the convex core of $H^{3} / \Gamma$, and consider the nearest point retraction $r: \Omega_{\Gamma} / \Gamma \rightarrow \partial C(\Gamma)$. Then $r$ is K-Lipschitz and has a homotopically inverse $K$-Lipschitz map.

The bottom spectrum $\lambda_{0}$ of Laplacian is the smallest eigenvalue of the Laplacian over the set of compactly supported smooth functions, $C_{0}^{\infty}(N)$, which is equal to

$$
\inf _{f \in C_{0}^{\infty}(N)}\left(\frac{\int_{N}|\nabla f|^{2}}{\int_{N} f^{2}}\right) .
$$

Equivalently it is the largest value of $\lambda$ for which there exists a positive $C^{\infty}$ function $f$ on $N$ such that $\triangle f+\lambda f=0$. Then it is clear that if $\tilde{N}$ covers $N$, then $\lambda_{0}(N) \leq \lambda_{0}(\tilde{N})$. Furthermore by Buser [8], see [12] for a complete argument, it is known that

Proposition 1. Let $N$ be an infinite volume, pinched negatively curved complete Riemannian manifold. Then

$$
\lambda_{0} \leq R \frac{\operatorname{Vol} C C(N)}{\operatorname{Vol} C(N)},
$$

where $R$ depends only on the dimension of the manifold and the curvature bounds.

Proof: If the Ricci curvature of a complete Riemannian $n$-manifold $N$ is bounded below by $-(n-1) \kappa^{2}$, then $\lambda_{0} \leq R \kappa h(N)$ where $R$ depends only on $n$ and $h(N)$ is the Cheeger constant which is defined to be the infimum, over all compact $n$-submanifolds $A$ of $N$, of $\frac{\operatorname{Vol}(\partial A)}{\operatorname{Vol}(A)}$. From this, the claim follows.

For a similar result, see [6]. For convex cocompact locally symmetric rank 1 manifold $X / \Gamma$, it is known that 
Lemma 2. $D(h-D) \leq \lambda_{0}(\Gamma)$ where $D$ is the Hausdorff dimension of the limit set $L_{\Gamma}$ and $h$ is the Hausdorff dimension of the boundary of $X$.

Proof: The function defined by $u(x)=\int e^{-D B(x, \theta)} d \mu_{0}(\theta)$ has $\triangle u=$ $D(h-D) u$, and so $D(h-D) \leq \lambda_{0}$.

For convex cocompact pinched negatively curved manifold $M$ it is known that the Hausdorff dimension of the limit set is equal to

$$
\lim _{R \rightarrow \infty} \frac{\log \#\left\{\gamma \mid l_{M}(\gamma) \leq R\right\}}{R}
$$

where $l_{M}(\gamma)$ is the length of the geodesic representative in the free homotopy class of $\gamma$. Also the critical exponent of the Poincare series is equal to the Hausdorff dimension of the limit set, see [47. So length spectrum determines the Hausdorff dimension of the limit set. For a Hadamard manifold $X$, if $\gamma$ is an isometry acting on $X$, the translation length $l_{X}(\gamma)$ of $\gamma$ is defined by

$$
\inf _{x \in X} d(x, \gamma x) .
$$

From these facts we obtain:

Theorem 4. Let $N=H^{3} / \Gamma$ be a convex cocompact, infinite volume hyperbolic 3-manifold, and let $D$ denote the Hausdorff dimension of the

limit set $L_{\Gamma}$. Then $D(2-D) \leq \lambda_{0} \leq C \frac{|\chi(\partial C(N))|}{\text { vol }(C(N))}$ for some universal constant $C$.

Proof: The inequality follows from Proposition 1 and Lemma 2.

\section{SOME EXAMPLES}

Mahler's criterion [17] states that if $G$ is a Lie group and $U$ is a neighborhood of the identity $e$, then the set of subgroups $\Gamma$ of $G$ such that $\Gamma \cap U=\{e\}$ is a compact set $\mathcal{U}_{G}$ with respect to the Chabauty topology. To see the relation between Chabauty topology and geometric topology in Gromov sense, see [2]. First observe the following useful fact [28], which will be used throughout the paper.

Lemma 3. Let $\Gamma$ be a fixed finitely generated group. Let $\rho: \Gamma \rightarrow G$ be a Zariski dense representation into a real semi-simple Lie group of rank 1. Then there is a finite generating set $\left\{\gamma_{1}, \cdots, \gamma_{k}\right\}$ depending only on $\Gamma$ so that the representation is determined up to conjugacy by the marked length spectrum on this finite generating set.

For a similar result in surface, see [9]. One can easily deduce the following. 
Proposition 2. Let $G$ be a semisimple Lie group of rank 1 with $X=$ $G / K$ and $\Lambda$ a discrete set of positive real numbers. Then the set of convex cocompact manifolds $\Gamma \backslash G / K$ with diameter for the convex core bounded above by $R$ and with a length spectrum without multiplicity contained in $\Lambda$, is finite up to isometry.

Proof: One can find a neighborhood $U$ of $e$ so that for any $\gamma \in U$, $l(\gamma)<r_{0}$ where $r_{0}$ is the smallest number in $\Lambda$. Suppose there exist infinitely many non-isometric convex cocompact manifolds $\Gamma_{i} \backslash G / K$ with its length spectrum contained in $\Lambda$. Since $\Gamma_{i} \in \mathcal{U}_{G}$, one can find $\Gamma$ so that $\Gamma_{i} \rightarrow \Gamma$ in Chabauty topology after passing to a subsequence. In a geometric term [2], it implies that, for $x_{i} \in C\left(X / \Gamma_{i}\right) \subset B\left(x_{i}, R\right)$,

$$
\left(X / \Gamma_{i}, x_{i}\right) \rightarrow(X / \Gamma, x)
$$

in Gromov sense, i.e., for any ball $B(x, r) \subset X / \Gamma$, there is a smooth embedding $f_{i}: B(x, r) \rightarrow X / \Gamma_{i}$ for large $i$ so that $f_{i}(x)=x_{i}$ and $f_{i}$ tends to an isometry in $C^{\infty}$ topology. Since $C\left(X / \Gamma_{i}\right) \subset B\left(x_{i}, R\right)$, for given small $\epsilon>0$, there exists $i_{0}$ such that for all $i>i_{0}, C\left(X / \Gamma_{i}\right) \subset$ $f_{i}(B(x, R+\epsilon))$. In negatively curved Hadamard manifolds, the convex core is a strong deformation retract of a convex cocompact manifold via shortest distance retraction to the convex core.

Then using $f_{j} \circ f_{i}^{-1}: f_{i}(B(x, R+\epsilon)) \rightarrow f_{j}(B(x, R+\epsilon))$ one can see that $C\left(X / \Gamma_{i}\right)$ are all homotopy equivalent to each other and so $\Gamma_{i}$ are all isomorphic to each other. Choose an isomorphism $\rho_{i}: \Gamma^{\prime} \rightarrow \Gamma_{i}$ induced from the map $f_{i}: B(x, R+\epsilon) \rightarrow X / \Gamma_{i}$, which induces a representation $\rho_{i}: \Gamma^{\prime} \rightarrow G$, where $\Gamma^{\prime}=\pi_{1}(x, B(x, R+\epsilon))$ is isomorphic to a subgroup of $\Gamma$. The surjectivity of $\rho_{i}$ is evident since any element in $\Gamma_{i}$ is represented by a loop based at $x_{i}$, which is contained in $C\left(X / \Gamma_{i}\right)$. Then the loop is realized by the image of some loop in $B(x, R+\epsilon)$ based at $x$ under $f_{i}$. The injectivity of $\rho_{i}$ follows from Gauss-Bonnet theorem

$$
\int_{D} K d A+\int_{\partial D} k_{g} d s=2 \pi \chi(D)=2 \pi
$$

where $K$ is Gaussin curvature of a disc $D$ and $k_{g}$ is a geodesic curvature of $\partial D$. Suppose $f_{i}(\gamma)$ represents a trivial element in $\pi_{1}\left(x_{i}, C\left(X / \Gamma_{i}\right)\right)$ for some geodesic loop $\gamma$ based at $x$, representing a non-trivial element in $\pi_{1}(x, B(x, R+\epsilon))$. Choose disc $D_{i}$ bounding $f_{i}(\gamma)$, whose Gaussian curvature $<-\delta$. Then for large $i>i_{0}$, since $f_{i}$ is almost an isometry, $f_{i}(\gamma)$ is almost a geodesic, hence $k_{g}$ along $\partial D_{i}$ is almost zero with the interior angle at $x_{i}$ is $\theta_{i}$. Hence the formula reads

$$
-\delta \operatorname{Area}\left(D_{i}\right)>2 \pi-\left(\pi-\theta_{i}\right)=\pi+\theta_{i},
$$

which gives $\operatorname{Area}\left(D_{i}\right)<\frac{\pi+\theta_{i}}{-\delta}<0$, a contradiction. 
Since $f_{i}$ is almost an isometry, it follows that $l_{\rho_{i}}(\gamma), \gamma \in \Gamma^{\prime}$ are bounded above. Since $\Lambda$ is a spectrum of a convex cocompact manifold, it is discrete. Then by passing to a subsequence, one can assume that $l_{\rho_{i}}(\gamma)$ is constant for a fixed $\gamma$. Fix a finite determining set $\left\{\gamma_{1}, \cdots, \gamma_{k}\right\}$ from Lemma 3. After passing to a subsequence we may assume that $l_{\rho_{i}}\left(\gamma_{j}\right)=l_{\rho_{l}}\left(\gamma_{j}\right), j=1, \cdots, k$ and for all $i, l$. But then $X / \Gamma_{i}$ are all isometric to each other, which contradicts the choice of $\Gamma_{i}$.

Note that a priori we did not assume that $\Gamma_{i}$ are isomorphic each other. That $\Gamma_{i}$ are isomorphic each other follows during the course of the proof. Also we did not use the multiplicity in the spectrum. In fact, the proof works even we just assume that the length spectrum without multiplicity is contained in a fixed discrete set, not necessarily the same. The issue for bounded diameter of convex core can be dealt with in real hyperbolic manifold as follows.

Corollary 1. Let $M$ be a hyperbolic 3-manifold with incompressible boundary and with a discrete length spectrum. Then there are finitely many convex cocompact hyperbolic 3-manifolds, up to isometry, homotopy equivalent to $M$ with the length spectrum $\Lambda(M)$ with multiplicity.

Proof: For convex cocompact manifold, $\Lambda(M)$ determines the critical exponent of Poincaré series which is the Hausdorff dimension of the limit set, $D$ as observed in section 2. Then Theorem 4 implies that the volume of the convex core is uniformly bounded above. Also for boundary incompressible manifold, the injectivity radius on convex core is uniformly bounded below by the smallest number in $\Lambda(M)$. So the diameter of the convex core has a uniform upper bound. Then the conclusion follows from Proposition 2 .

In the next section, we will prove a theorem without multiplicity condition using Kleinian group theory.

\section{Hyperbolic 3-Manifold With InCOMPRESSIBle Boundary}

Let $M$ be a fixed hyperbolic 3-manifold. Then $A H(M)$ denotes the set of marked hyperbolic 3-manifolds homotopy equivalent to $M$. This is the set of discrete faithful representations from $\pi_{1}(M)$ to $\operatorname{PSL}(2, \mathbb{C})$ up to conjugacy. In this reason one denotes it by $A H\left(\pi_{1}(M)\right)$. Hence an element in $A H(M)$ can be written as $N=(f, M, N)$ with

$$
f: M \rightarrow N
$$

where $f$ is a marking and $N$ is a hyperbolic 3-manifold homotopy equivalent to $M$. 
From now on, we describe some convergent theorem for hyperbolic 3-manifolds. Indeed, from [15], one can extract an algebraically convergent subsequence if a sequence $N_{i}$ has a lower bound for injectivity radius. For the readers who are not familiar with 3 -dimensional hyperbolic geometry, we give some ideas.

The window $W$ of $M$ consists of the $I$-bundle components of the characteristic submanifold $\Sigma(M)$ together with a thickened neighborhood of every essential annulus in $\partial \Sigma(M) \backslash \partial M$ which is not the boundary of an $I$-bundle component of $\Sigma(M)$. The window itself is an $I$-bundle over a surface $w$, which is called the window base.

Thurston [46] showed that if $\Gamma$ is any subgroup of $\pi_{1}(M)$ which is conjugate to the fundamental group of a component of $M \backslash W$ whose closure is not a thickened torus, then a restriction of any sequence in $A H\left(\pi_{1}(M)\right)$ to $A H(\Gamma)$ has a convergent subsequence. Using this they proved the following [15]:

Theorem 5. Let $\left\{\rho_{i}\right\}$ be a sequence in $A H\left(\pi_{1}(M)\right)$. We may then find a subsequence $\left\{\rho_{j}\right\}$, a sequence of elements $\left\{\phi_{j}\right\}$ of Out $\left(\pi_{1}(M)\right)$, and a collection $x$ of disjoint, non-parallel, homotopically non-trivial simple closed curves in the window base $w$ such that if $\Gamma$ is any subgroup of $\pi_{1}(M)$ which is conjugate to the fundamental group of a component of $M \backslash X$ whose closure is not a thickened torus, where $X$ is the total space of the I-bundle over $x$, then $\left\{\left.\rho_{j} \circ \phi_{j}\right|_{\Gamma}\right\}$ converges in $A H(\Gamma)$. Moreover, if $c$ is a curve in $x$, then $\left\{l_{\rho_{j} \circ \phi_{j}}(c)\right\}$ converges to 0 .

Specially if $\rho_{i} \in A H\left(\pi_{1}(M)\right)$ is a sequence with lower bound on injectivity radius, then there exists a subsequence $\rho_{j}$ and $\phi_{j} \in \operatorname{Out}\left(\pi_{1}(M)\right)$ so that $\rho_{j} \circ \phi_{j}$ converges algebraically.

The idea is as follows. By Thurston, the restrictions of the representations to the complementary components of the window have convergent subsequences, and the lengths of the window boundaries are bounded. For each $\rho_{i}$, represent $w$ as a pleated surface with geodesic boundary. After passing to a subsequence and remarkings, either the hyperbolic structures induced by pleated surfaces converge or develop cusps. In the latter case we cut along the curves which become cusps (these are the family $x$ ), and argue that the representations restricted to the remaining components converge up to subsequence. Finally, one reglues along the window boundaries which did not converge to cusps, and after passing to further subsequence obtain such a convergent subsequence.

Now we prove the following theorem.

Theorem 6. Let $M$ be a hyperbolic 3-manifold with incompressible boundary and $\Lambda$ a discrete subset of $\mathbb{R}$. Then there are finitely many 
hyperbolic 3-manifolds, up to isometry, homotopy equivalent to $M$ so that the length spectrum without multiplicity is contained in $\Lambda$.

Proof: Let $N_{i}=H^{3} / \rho_{i}\left(\pi_{1}(M)\right)$ be infinitely many non-isometric such hyperbolic manifolds whose length spectrum without multiplicity is contained in $\Lambda$.

Suppose after passing to a subsequence, we can obtain a convergent sequence $\left\{\rho_{i} \circ\left(\phi_{i}\right)_{*}\right\}$ converging to $\rho$ up to changing the marking by $\phi_{i}$, which is guaranteed by Theorem 5 .

We have

$$
l_{\rho_{i} \circ\left(\phi_{i}\right)_{*}}(\gamma) \rightarrow l_{\rho}(\gamma)
$$

Since $\left\{l_{\rho_{i} \circ\left(\phi_{i}\right)_{*}}(\gamma) \subset \Lambda\right\}$ is discrete, after passing to a subsequence, we conclude that $l_{\rho_{i} \circ\left(\phi_{i}\right)_{*}}(\gamma)=l_{\rho}(\gamma)$ for a fixed $\gamma$. Then one can repeat this for a finite set of $\gamma_{1}, \cdots, \gamma_{n}$ in $\pi_{1}(M)$. By Lemma 3 or by [26], a representation is determined up to conjugacy by the translation length of a finitely many elements in $\pi_{1}(M)$. So we conclude that $\rho_{i} \circ\left(\phi_{i}\right)_{*}$ represent isometric manifolds, which is a contradiction.

For future purpose, we record the following theorem as well [36].

Theorem 7. Let $\rho_{i}: \Gamma \rightarrow P S L(2, \mathbb{C})$ be a sequence of representations such that $l_{\rho_{i}}(\gamma)$ is uniformly bounded for each $\gamma \in \Gamma$. Then there is a convergent subsequence.

\section{HyPERBoliC 3-MANIFOLD WhOSE INJECTIVITY RADIUS IS BOUNDED FROM BELOW ON CONVEX CORE}

In this section we discard the assumption that $M$ has an incompressible boundary, nonetheless we stick to the case where manifolds are convex cocompact.

Now we state the theorem. Note that when $M$ has a compressible boundary, the smallest geodesic length of $M$ does not determine the injectivity radius on the convex core due to the compression disk.

Theorem 8. Suppose $M$ is a convex cocompact hyperbolic 3-manifold. Then the set of convex cocompact hyperbolic 3-manifolds homotopy equivalent to $M$ with the length spectrum $\Lambda(M)$ and with the injectivity radius of the convex core being bounded from below in the sense of Definition 1, is finite up to isometry.

Proof: Suppose there are infinitely many non-isometric convex cocompact hyperbolic 3-manifolds $N_{i}$ with $\Lambda(M)$. We will derive a contradiction as before. The unmarked length spectrum determines a critical exponent of Poincaré series which is equal to $D$ in convex cocompact case. Then by Theorem 4 , the volume of the convex core has an upper 
bound. Since the injectivity radius of the convex core is bounded from below, its diameter has an upper bound. This can be easily seen as follows. By the defition of injectivity radius, $\cup B_{r}\left(x_{i}\right) \cup B_{r}\left(y_{j}\right)$ covers $C\left(N_{i}\right)$ with the condition $d\left(x_{i}, x_{j}\right)>r, d\left(x_{i}, y_{j}\right)>r, d\left(y_{i}, y_{j}\right)>r$. Set $\operatorname{vol}\left(B_{r}(x)\right)=\epsilon$. Since $x_{i}$ and $y_{j}$ are $r$-separated one can easily estimate

$$
\operatorname{vol}\left(\cup B_{r}\left(x_{i}\right) \cup B_{r}\left(y_{j}\right)\right) \geq f(\text { number of balls }) \epsilon
$$

where $f$ (number of balls) $\rightarrow \infty$ as the number of balls goes to infinity. Hence the number of balls to cover $C\left(N_{i}\right)$ is uniformly bounded and hence the diameter is uniformly bounded.

Take a base point $p_{i} \in C\left(N_{i}\right)$. Then the injectivity radius at $p_{i}$ is bounded below by the assumption. Then the sequence $\left(N_{i}, p_{i}\right)$ converge to $(N, p)$ in geometric topology where $N$ is a complete hyperbolic 3-manifold, [35]. Take a number $R$ which is greater than the diameter of $C\left(N_{i}\right)$ for all $i$. By the definition of geometric convergence, there exist a ball $B(p, R) \subset N$ and a smooth embedding $f_{i}$ : $B(p, R) \rightarrow N_{i}$ whose quasiisometric constant converges to 1 as $i$ tends to $\infty$, and so $f_{i}(B(p, R))$ contains $C\left(N_{i}\right)$ and $B(p, R)$ is homeomorphic to a neighborhood of $C\left(N_{i}\right)$ for all large $i$. This can be easily seen as follows. As in the proof of Proposition 2, one can show $\left(f_{i}\right)_{*}: \pi_{1}(p, B(p, R)) \rightarrow \pi_{1}\left(p_{i}, f_{i}(B(p, R))\right)$ is an isomorphism. And since $C\left(N_{i}\right) \subset f_{i}(B(p, R))$, the nearest point retraction of $f_{i}(B(p, R))$ to $C\left(N_{i}\right)$ induces a homotopy equivalence between $B(p, R)$ and $C\left(N_{i}\right)$. Since a neighborhood of $C\left(N_{i}\right)$ is homeomorphic to $N_{i}$, we conclude that $N_{i}$ are all homeomorphic to $B(p, R)$. Note that $B(p, R)$ is homotopy equivalent to $M$.

Now we can argue as in the proof of Proposition 2, Let $\gamma_{1}, \cdots, \gamma_{k} \in$ $\pi_{1}(M)$ be a determining set, i.e., the marked length spectrum of these elements determines the representation up to conjugacy.

Now give a marking $f_{i}: B(p, R) \rightarrow N_{i}$ to $N_{i}$, which will induce a representation $\rho_{i}: \pi_{1}(M) \rightarrow P S L(2, \mathbb{C})$ corresponding to $N_{i}$. Then $l_{N_{i}}\left(\gamma_{1}\right) \rightarrow l_{N}\left(\gamma_{1}\right)$. Since $\Lambda(M)$ is discrete, after passing to a subsequence, we may assume that $l_{N_{i}}\left(\gamma_{1}\right)=l_{N}\left(\gamma_{1}\right)$. Doing this $k$-times, we get $l_{N_{i}}\left(\gamma_{j}\right)=l_{N}\left(\gamma_{j}\right)$ for all $i$ and $j=1, \cdots, k$. This shows that all $N_{i}$ are isometric to each other, which is a contradiction.

\section{Hyperbolic 3-MANifold Without FREE FACTORS}

In this section we prove our main result. Let $M$ be a convex cocompact hyperbolic 3-manifold with compressible boundary which does not have a handlebody factor, i.e., it has incompressible cores $M_{i}$ such that a unique 1-handle $h^{i}$ connects $M_{i}$ to $M_{i+1}$. In terms of the fundamental 


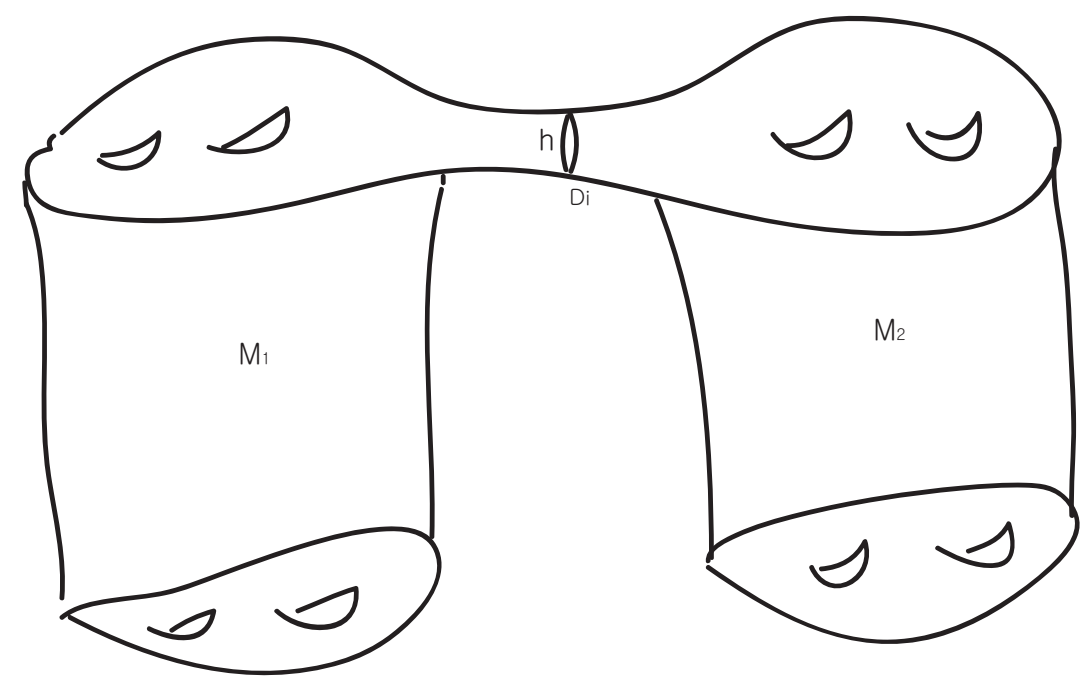

Figure 1. $M$ consists of $M_{1}, M_{2}$ and one handle $h$.

group, $\pi_{1}(M)$ does not have a free factor $F_{n}$ where $F_{n}$ is a free group with $n$ generators, see section 2 .

Theorem 9. Let $M$ be a convex cocompact real hyperbolic 3-manifold (which is not a solid torus) with a length spectrum $\Lambda$ with multiplicity. Suppose $\pi_{1}(M)$ does not have any free factor, i.e., $\pi_{1}(M)$ cannot be represented as $G * F_{n}$ where $F_{n}, n \geq 1$ is a free group of $n$-generators. Then there exists only finitely many non-isometric convex cocompact hyperbolic 3-manifolds which are homotopy equivalent to $M$ and with the length spectrum $\Lambda(M)$ with multiplicity.

Proof: Suppose there is an infinite sequence of mutually non-isometric convex cocompact hyperbolic 3-manifolds $N_{i}=H^{3} / \Gamma_{i}$ with length spectrum $\Lambda(M)$. We will first deal with the case where there is only one 1-handle to give a better understanding to the reader. First assume that the incompressible core of $M$ is $M_{1} \cup M_{2}$ and $M$ is obtained from this by adding an 1-handle $h$ glued to boundaries of $M_{1}$ and $M_{2}\left(M_{1}=M_{2}\right.$ is not allowed). Then $\pi_{1}(M)=\pi_{1}\left(M_{1}\right) * \pi_{1}\left(M_{2}\right)$. Accordingly $\Gamma_{i}=\Gamma_{i}^{1} * \Gamma_{i}^{2}$. Let $N_{i}^{j}$ be a cover of $N_{i}$ corresponding to $\pi_{1}\left(M_{j}\right), j=1,2$. Since $M_{1}$ has incompressible boundary, $N_{i}^{1}$ has incompressible boundary also. Note that $N_{i}^{1}$ is convex cocompact by covering theorem [14]. Now we apply Theorem 6. Since $\Lambda\left(N_{i}^{j}\right) \subset \Lambda\left(N_{i}\right)=\Lambda(M)$, after passing to a subsequence, we may assume that all $N_{i}^{1}$ are isometric to each other. The same thing is true for $N_{i}^{2}$. 
By using a covering map $P_{j}: N_{i}^{j} \rightarrow N_{i}$ (we drop $i$ in $P_{j}$ for simplicity), $N_{i}$ has two parts $P_{1}\left(C\left(N_{i}^{1}\right)\right)$ and $P_{2}\left(C\left(N_{i}^{2}\right)\right)$ homotopy equivalent to $M_{1}$ and $M_{2}$ respectively, such that $N_{i}^{j}$ are isometric to each other for all $i$ and fixed $j=1,2$. Note that $P_{1}\left(C\left(N_{i}^{1}\right)\right)$ and $P_{2}\left(C\left(N_{i}^{2}\right)\right)$ might not be disjoint. Since the convex hull is the smallest convex set containing all the closed geodesics, it is clear that $P_{j}\left(C\left(N_{i}^{j}\right)\right) \subset C\left(N_{i}\right)$. For each closed geodesic $\gamma \subset N_{i}^{j}, P_{j}: \gamma \rightarrow P_{j}(\gamma)$ is a homeomorphism of degree 1 viewed as a self map from a circle to itself since $N_{i}^{j}$ is a covering corresponding to $\pi_{1}\left(M_{j}\right)$. In particular geodesic lengths coming from $P_{1}\left(C\left(N_{i}^{1}\right)\right)$ are all the same for all $i$, and the same thing is true for $P_{2}\left(C\left(N_{i}^{2}\right)\right)$.

Since $C\left(N_{i}\right)$ is homotopy equivalent to $M$, decompose $C\left(N_{i}\right)=$ $C_{i}^{1} \cup h \cup C_{i}^{2}$ such that $C_{i}^{1}$ contains $P_{1}\left(C\left(N_{i}^{1}\right)\right)$, convex and homotopy equivalent to $M_{1}, C_{i}^{2}$ contains $P_{2}\left(C\left(N_{i}^{2}\right)\right)$, convex and homotopy equivalent to $M_{2}$, and $h$ is a 1-handle joining them. Note here that $C_{i}^{1}, h$ and $C_{i}^{2}$ are not mutually disjoint in general. Since $C_{i}^{j}$ has irreducible boundary and the length spectrum is fixed for it, the injectivity radius is bounded below on $C_{i}^{j}$. Hence by Theorem 4 again, the diameter of $C_{i}^{j}$ is bounded.

If the injectivity radius of $C\left(N_{i}\right)$ in the sense of Definition 1 is bounded below, then we are done by Theorem 8 , hence assume that the injectivity radius goes to zero as $i \rightarrow \infty$. This is possible only along compression disks which are the dual core of attached one-handles since the injectivity radius is bounded below on $C_{i}^{j}$.

Let the length of the meridian, which is the boundary of compression disk $D_{i}$, tend to zero as $i \rightarrow \infty$. See Figure 1 . Then by Theorem 3, the meridian length in corresponding conformal boundary tends to zero also. By the proof of Lemma 1, there exists a solid cylinder neighborhood of $D_{i}$ whose height tends to infinity. Hence $h$ is non-empty and the length of any geodesic crossing $h$ goes to infinity.

Find a geodesic $\gamma$ which is not in $P_{1}\left(C\left(N_{1}^{1}\right)\right)$, neither in $P_{2}\left(C\left(N_{1}^{2}\right)\right)$. Then $\gamma$ must cross $h$. Take $i$ large enough so that the length in $N_{i}$ of any geodesic crossing the 1-hanlde $h$ is much greater than $l_{N_{1}}(\gamma)$. This is possible by Lemma 1 as explained above. Now by assumption, $N_{i}$ and $N_{1}$ have the same length spectrum with multiplicity. Note also that $P_{j}\left(C\left(N_{i}^{j}\right)\right)$ and $P_{j}\left(C\left(N_{1}^{j}\right)\right), j=1,2$ have the same length spectrum since $N_{i}^{j}$ are isometric. If $l_{N_{1}}(\gamma)$ is the length of a geodesic in $N_{1}^{j}$, the multiplicity for $l_{N_{1}}(\gamma)$ will be different for $N_{1}$ and $N_{i}$. If $l_{N_{1}}(\gamma)$ is not the length of any geodesic in $N_{1}^{j}$, there will be no geodesic in $N_{i}$ whose length is equal to $l_{N_{1}}(\gamma)$. In either case, $N_{1}$ and $N_{i}$ do not have the same length spectrum. Note that we are considering the 
length spectrum with multiplicity. Hence the injectivity radius on $C\left(N_{i}\right)$ should remain bounded below, and we are done by Theorem 8 ,

Now we deal with the case where there are many 1-handles. For a given $M$, suppose $M=M_{1} \cup h^{1} \cup M_{2} \cup h^{2} \cup \cdots \cup h^{t} \cup M_{t+1}$. By taking a cover $N_{i}^{k}$ corresponding to $M_{k}$, by Theorem [6] after passing to a subsequence, we may assume that all $N_{i}^{k}$ are isometric to each other for a fixed $k$. Now using the projection $P_{k}: N_{i}^{k} \rightarrow N_{i}$, there are two cases to consider. If the injectivity radius is bounded below on $C\left(N_{i}\right)$, we are done by Theorem 8. As before, decompose $C\left(N_{i}\right)=$ $C_{i}^{1} \cup h_{i}^{1} \cup C_{i}^{2} \cup h_{i}^{2} \cup C_{i}^{3} \ldots$ such that $C_{i}^{l}$ contains $P_{l}\left(C\left(N_{i}^{l}\right)\right)$, convex and homotopy equivalent to $M_{l}$. Note that the injectivity radius on $C_{i}^{k}$ is uniformly bounded below.

If the injectivity radius on $C\left(N_{i}\right)$ is not bounded below, choose the first $h_{i}^{l}$ whose height tends to infinity as in the previous case.

Lemma 4. Let $M^{\prime}=M_{1} \cup h^{1} \cup \cdots \cup h^{l-1} \cup M_{l}$ and $N_{i}^{\prime}$ the corresponding covering manifolds. Then $N_{i}^{\prime}$ are isometric to each other after passing to a subsequence.

Proof. By construction, all the 1-handles in $C\left(N_{i}^{\prime}\right)$ have bounded lengths. Any element $\gamma$ in $\pi_{1}\left(M^{\prime}\right)=\pi_{1}\left(M_{1}\right) * \cdots * \pi_{1}\left(M_{l}\right)$ can be written as $\gamma_{1} * \cdots * \gamma_{l}$ where base points for $\gamma_{i}$ can be connected by arcs going over 1-handles. Since 1-handles have bounded lengths and $N_{i}^{k}$ are all isometric to each other, each $\gamma_{i}$ is realized as a geodesic which has the same length for all $i$, and the arcs connecting them have the bounded lengths. This implies that any $\gamma$ in $\pi_{1}\left(M^{\prime}\right)$ can be realized as a geodesic which has a uniform bounded length for all $i$. Then by Theorem 7 , the representations $\rho_{i}: \pi_{1}\left(M^{\prime}\right) \rightarrow P S L(2, \mathbb{C})$ corresponding to $N_{i}^{\prime}$ converge to some representation $\rho$ after passing to a subsequence. Since the length spectrum of $N_{i}^{\prime}$ is contained in a fixed discrete length spectrum, using the same argument as in the proof of Theorem [6] on a finitely many determining elements in $\pi_{1}\left(M^{\prime}\right)$, after passing to a subsequence, $N_{i}^{\prime}$ are all isometric to each other.

In this way collect submanifolds $M_{1}^{\prime}, \cdots, M_{k}^{\prime}$ so that $M=M_{1}^{\prime} \cup h^{1} \cup$ $\cdots \cup h^{k-1} \cup M_{k}^{\prime}$ and the handles in $M_{m}^{\prime}, m=1, \cdots, k$ have bounded lengths throughout the sequence, and the other handles are getting longer as $i \rightarrow \infty$. Then after passing to a subsequence, all the coverings $N_{m, i}^{\prime}, m=1, \cdots, k$ of $N_{i}$ corresponding to $M_{m}^{\prime}$ are isometric to each other by Lemma 4, Let $P_{m}: N_{m, i}^{\prime} \rightarrow N_{i}$ be a covering map, and decompose

$$
C\left(N_{i}\right)=C_{i}^{1} \cup h_{i}^{1} \cup \cdots \cup h_{i}^{k-1} \cup C_{i}^{k}
$$


where $C_{i}^{m}$ is a convex set containing $P_{m}\left(C\left(N_{m, i}^{\prime}\right)\right)$ homotopy equivalent to $M_{m}^{\prime}$. Note that the length of $h_{i}^{m}$ goes to infinity as $i \rightarrow \infty$ and the length spectrum on $C_{i}^{m}$ is fixed for all $i$ since $N_{m, i}^{\prime}$ are all isometric to each other.

Now apply the same logic as in the case where there exists only one 1handle as follows. Choose $\gamma$ passing through $h^{1}$. Choose $i$ large enough so that any loop passing through one of the handles has length in $N_{i}$ greater than $\ell_{N_{1}}(\gamma)$. This is possible since all the lengths of handles $h_{i}^{m}, m=1, \cdots, k$, go to infinity. If $\ell_{N_{1}}(\gamma)$ is realized as a length of some geodesic in $C_{1}^{m}$, the multiplicity in $N_{1}$ for $\ell_{N_{1}}(\gamma)$ is greater than the multiplicity in $N_{i}$. If $\ell_{N_{1}}(\gamma)$ is not realized as a length of any curve in $C_{1}^{m}$ for all $m=1, \cdots, k$, this length is not realized in $N_{i}$ at all. In any case, $N_{1}$ and $N_{i}$ will have different length spectrums with multiplicity, which is a contradiction.

Hence, there is no handle whose length goes to infinity. This implies that the injectivity radius of $C\left(N_{i}\right)$ is bounded below. Then by Theorem 8, we are done.

Remark Somehow handlebody case is difficult to handle. If two generators are getting longer and their invariant axes are getting closer, it is hard to draw a contradiction.

\section{RANK ONE SYMMETRIC SPACE CASE}

In this section we prove the isospectral finiteness of surface group in rank one symmetric space. It is known that one can deform a totally real hyperbolic surface sitting in complex, quaternionic and octonionic hyperbolic manifold [27]. If the deformation is small, the group becomes convex cocompact.

Let $M$ and $N$ be Riemannian manifolds of dimension $m, n$ respectively with metric tensors $\left(\gamma_{\alpha \beta}\right)$ and $\left(g_{i j}\right)$. The curvature tensor is defined by

$$
R(X, Y) Z=\nabla_{X} \nabla_{Y} Z-\nabla_{Y} \nabla_{X} Z-\nabla_{[X, Y]} Z
$$

and the sectional curvature spanned by orthonormal $X, Y$ is

$$
K(X, Y)=\langle R(X, Y) Y, X\rangle .
$$

Let $f: M \rightarrow N$ be a $C^{1}$ map and using local charts $\left(x^{1}, \cdots, x^{m}\right)$ on $M,\left(f^{1}, \cdots, f^{n}\right)$ on $N$, the energy density is

$$
e(f)(x)=\frac{1}{2} \gamma^{\alpha \beta}(x) g_{i j}(f(x)) \frac{\partial f^{i}(x)}{\partial x^{\alpha}} \frac{\partial f^{j}(x)}{\partial x^{\beta}} .
$$


If $\left\{e_{1}, \cdots, e_{m}\right\}$ is an orthonormal basis of $T_{x} M$, then

$$
e(f)(x)=\frac{1}{2} \sum\left\langle d f\left(e_{i}\right), d f\left(e_{i}\right)\right\rangle=\frac{1}{2}|d f|^{2} .
$$

The energy of $f$ is

$$
E(f)=\int_{M} e(f) d v o l_{M}
$$

A solution of the Euler-Lagrange equation for $E$ is called a harmonic map.

The Laplace-Beltrami operator $\triangle$ is defined so that

$$
\triangle f=-\sum \frac{\partial^{2} f}{\left(\partial x^{i}\right)^{2}}
$$

for real valued function $f$.

Bochner type equality for a harmonic map $f: M \rightarrow N$ is [25]

$$
\begin{gathered}
-\triangle e(f)(x) \\
=|\nabla d f|^{2}+\left\langle d f \operatorname{Ric}\left(e_{i}\right), d f\left(e_{i}\right)\right\rangle-\left\langle R_{N}\left(d f\left(e_{i}\right), d f\left(e_{j}\right)\right) d f\left(e_{j}\right), d f\left(e_{i}\right)\right\rangle .
\end{gathered}
$$

Here $\left\{e_{i}\right\}$ is an orthonormal basis of $T_{x} M$ and

$$
\operatorname{Ric}(v)=\sum R_{M}\left(v, e_{i}\right) e_{i} .
$$

Let $(S, \sigma)$ be a hyperbolic surface and $G$ a semisimple Lie group, $X$ the associated symmetric space. Let $\rho: \pi_{1}(S) \rightarrow G$ be a representation whose image is reductive. Let $J$ be a complex structure compatible with $\sigma$ on $(S, \sigma)$ lifted to $\tilde{S}$ and $f: \tilde{S} \rightarrow X$ a smooth $\rho$-equivariant map. Then

$$
(d f \wedge d f \circ J)(u, v)=\frac{1}{2}(\langle d f(v), d f(J u)\rangle-\langle d f(u), d f(J v)\rangle)
$$

defines an exterior differential 2 -form on $\tilde{S}$, which descends to $S$. The energy of $f$ with respect to $J$ (or $\sigma$ ) can be alternatively defined by

$$
E(J, f)=\int_{S} d f \wedge d f \circ J
$$

Then the energy functional $E_{\rho}$ on $\mathcal{T}(S)$ is defined by

$$
E_{\rho}(J)=\inf _{f} E(J, f)
$$

for all $\rho$-equivariant $f$.

Then there exists a $\rho$-equivariant harmonic map $f:(\tilde{S}, \tilde{\sigma}) \rightarrow X$ inducing $\rho$ with energy $E_{\rho}(\sigma)$, see [18]. This function $E_{\rho}$ is a proper function on $\mathcal{T}(S)$ if $\rho$ is convex cocompact [30]. So there is a harmonic map $f_{\rho}$ and a hyperbolic metric $\sigma_{\rho}$ so that

$$
f_{\rho}:\left(\tilde{S}, \tilde{\sigma}_{\rho}\right) \rightarrow X
$$


minimizes the energy for all such possible choices of $f$ and $\sigma$. This map is known to be conformal [41].

Theorem 10. Let $G$ be a semisimple real Lie group of rank one of noncompact type. Fix $\Lambda$ a discrete set of positive real numbers, and a closed surface $S$ of genus $\geq 2$. Then the set of convex cocompact representations $\rho: \pi_{1}(S) \rightarrow G$ with $\Lambda_{\rho}=\Lambda$ is finite up to conjugacy and the change of marking. Here $\Lambda_{\rho}$ is the set of translation lengths of $\rho\left(\pi_{1}(S)\right)$.

Proof: Suppose there are infinitely many non-conjugate representations $\rho_{i}: \pi_{1}(S) \rightarrow G$ with $\Lambda_{\rho_{i}}=\Lambda$. Choose a harmonic map $h_{i}$ : $\left(\tilde{S}, \sigma_{i}\right) \rightarrow X$ so that the energy is the smallest among all such $h_{i}$ inducing $\rho_{i}$ and among all hyperbolic metrics $\sigma_{i}$. Then it is known that [41], $h_{p}$ is conformal, i.e., $d h_{p}\left(e_{1}\right)$ and $d h_{p}\left(e_{2}\right)$ are orthogonal and have the same norm for an orthonomal basis $\left\{e_{1}, e_{2}\right\}$ of $T_{x}\left(S, \sigma_{p}\right)$. Hence $\left|d h_{p}\right|^{2}=2\left|d h_{p}\left(e_{1}\right)\right|^{2}$. Then by Bochner formula

$$
\begin{gathered}
-\frac{1}{2} \triangle\left|d h_{p}\right|^{2}=\left|\nabla d h_{p}\right|^{2} \\
+\left\langle d h_{p} R i c_{\sigma_{p}}\left(e_{i}\right), d h_{p}\left(e_{i}\right)\right\rangle-\left\langle R_{X}\left(d h_{p}\left(e_{i}\right), d h_{p}\left(e_{j}\right)\right) d h_{p}\left(e_{j}\right), d h_{p}\left(e_{i}\right)\right\rangle,
\end{gathered}
$$

where $e_{1}, e_{2}$ is an orthonormal basis at a point of $\left(S, \sigma_{p}\right)$.

Since

$$
\operatorname{Ric}\left(e_{1}\right)=R\left(e_{1}, e_{1}\right) e_{1}+R\left(e_{1}, e_{2}\right) e_{2}
$$

and since

$$
\left\langle\operatorname{Ric}\left(e_{1}\right), e_{1}\right\rangle=\left\langle R\left(e_{1}, e_{2}\right) e_{2}, e_{1}\right\rangle=-1,
$$

and similar for $\operatorname{Ric}\left(e_{2}\right)$, we get $\operatorname{Ric}\left(e_{i}\right)=-e_{i}+x_{i} e_{j}$ to have

$$
\begin{gathered}
-\frac{1}{2} \Delta\left|d h_{i}\right|^{2} \\
\geq-\left|d h_{i}\right|^{2}-\left|d h_{i}\left(e_{1}\right)\right|^{4} 2 K_{X}\left(\frac{d h_{i}\left(e_{1}\right)}{\left|d h_{i}\left(e_{1}\right)\right|}, \frac{d h_{i}\left(e_{2}\right)}{\left|d h_{i}\left(e_{1}\right)\right|}\right),
\end{gathered}
$$

where $X$ is an associated symmetric space. Since $X$ is a rank one symmetric space, $K_{X}\left(\frac{d h_{i}\left(e_{1}\right)}{\left|d h_{i}\left(e_{1}\right)\right|}, \frac{d h_{i}\left(e_{2}\right)}{\left|d h_{i}\left(e_{1}\right)\right|}\right) \leq-1$. This implies that

$$
-\frac{1}{2} \triangle\left|d h_{i}\right|^{2} \geq\left|d h_{i}\right|^{2}\left(\left|d h_{i}\left(e_{k}\right)\right|^{2}-1\right)
$$

for $e_{k}, k=1,2$. If $x_{0} \in S$ is a maximum point of $\left|d h_{i}\right|^{2}$, then

$$
-\triangle\left|d h_{i}\right|^{2}\left(x_{0}\right) \leq 0
$$

so we get

$$
\left|d h_{i}\left(e_{k}\right)\right|^{2} \leq 1
$$


which implies that

$$
\left|d h_{i}(v)\right| \leq|v|
$$

for $v \in T_{x_{0}} S$. For any $x \in S$, since

$$
2\left|d h_{i}(x)\left(e_{k, x}\right)\right|^{2}=\left|d h_{i}(x)\right|^{2} \leq\left|d h_{i}\left(x_{0}\right)\right|^{2}=2\left|d h_{i}\left(x_{0}\right)\left(e_{k, x_{0}}\right)\right|^{2} \leq 2
$$

for $k=1,2$, we get

$$
l_{\rho_{i}}(\gamma) \leq l_{\sigma_{i}}(\gamma)
$$

for all $\gamma \in \pi_{1}(S)$. But since $l_{\rho_{i}}(\gamma) \geq r_{0}$ where $r_{0}$ is the smallest number in $\Lambda$, using Mumford compactness theorem, we get, after changing the marking of $\left(S, \sigma_{i}\right),\left(S, \sigma_{i}\right) \rightarrow(S, \sigma)$. Then after changing the marking of $\rho_{i}$ accordingly, we get

for all $\gamma \in \pi_{1}(S)$.

$$
l_{\rho_{i}}(\gamma) \leq C l_{\sigma}(\gamma)
$$

Then one can pass to a subsequence so that $\rho_{i} \rightarrow \rho$. Then

$$
l_{\rho_{i}}(\gamma) \rightarrow l_{\rho}(\gamma)
$$

for all $\gamma \in \pi_{1}(S)$. By discreteness of $\Lambda$, for each $\gamma$, we may assume that $l_{\rho_{i}}(\gamma)=l_{\rho}(\gamma)$ for large $i$. We repeat this for finitely many $\left\{\gamma_{1}, \cdots, \gamma_{n}\right\}$ which determines the representations in $G$ up to conjugacy by Lemma 3. So we conclude that $\rho_{i}$ and $\rho$ are conjugate for all $i$, which is forbidden by the assumption.

\section{Appendix A}

The materials covered in this appendix are well-known to the experts, specially for dimension 3 it is classical, though it is difficult to find a right literature for higher dimension, but for the convenience of the readers we give some proofs. The goal of this appendix is to show that the hyperbolic metric is uniquely determined by the metrics on the smooth convex boundary, Theorem 13, In this section $H_{\mathbb{R}}^{n}=H^{n}$.

A homeomorphism $\phi: X \rightarrow Y$ is $K$-quasiconformal if $\phi$ has distributional first derivatives locally in $L^{n}$, and

$$
\frac{1}{K}|\operatorname{det} D \phi(x)| \leq\left(\frac{|D \phi(v)|}{|v|}\right)^{n} \leq K|\operatorname{det} D \phi(x)|
$$

for almost every $x$ and every nonzero vector $v \in T_{x} X$. It is well-known from the techniques of Mostow rigidity that any quasiisometry from $H^{n}$ to itself extends to a quasiconformal map from the ideal boundary $S^{n-1}$ to itself. Also it is clear that if the extended map is a conformal map, then the original map is isotopic to an isometry.

Here we use a technique of Sullivan [42]. The action of a discrete group $\Gamma$ on a measure space is conservative if there is no positive measure set $A$ so that the translates $\{\gamma(A): \gamma \in \Gamma\}$ are disjoint up to 
measure zero set. In our case, we are using a Lebesque measure on $S^{n-1}$. The following is due to Sullivan.

Theorem 11. A discrete torsion free subgroup of $I$ so $\left(H^{n}\right)$ admits no invariant $k$-plane field $(0<k<n-1)$ on the part of $S_{\infty}^{n-1}$ where its action is conservative.

A direct proof can be found in [35].

First we prove the following observation.

Lemma 5. Suppose $\Gamma$ is a discrete group of $I s o\left(H^{n}\right)$. If the convex core of $\Gamma$ has an upper bound on the injectivity radius, then $\Gamma$ acts conservatively on its limit set $L_{\Gamma}$.

Proof: Actually this statement is proved in Theorem 5.11 of [31] for $H^{3}$ and the same proof works for any dimension $\geq 3$, but for reader's convenience we sketch the proof. Suppose not. Then there is a positive measure set $A \subset L_{\Gamma}$ so that $\{\gamma(A): \gamma \in \Gamma\}$ are disjoint up to measure zero sets. Then it is not difficult to see that for a Dirichlet polyhedron $P_{a}=\left\{p \in H^{n} \mid d(p, a) \leq d(p, \gamma(a))\right.$ for any $\left.\gamma \in \Gamma\right\}, \overline{P_{a}} \cap L_{\Gamma}$ has positive measure. Then a geodesic ray from $a$ to a Lebesgue density point in $\overline{P_{a}} \cap L_{\Gamma}$, which is contained in $P_{a}$ and the convex hull of $L_{\Gamma}$, has unbounded distance to $\partial P_{a}$. This shows that along this geodesic ray, the injectivity radius tends to infinity since $P_{a}$ is a fundamental domain of $\Gamma$.

For dimension 3, due to the solution of Ahlfors conjecture [1, 10], the limit set of a finitely generated Kleinian group has either zero measure or whole measure. If it has zero measure, the action on the limit set is conservative automatically, if whole measure, the limit set is $S^{2}$ and the action is ergodic, so conservative. See [13]. But as far as we know, Ahlfors conjecture is not known for dimension $\geq 4$.

From these two facts we will derive a useful conclusion. For dimension 3, it is a classical result by Ahlfors-Bers theory. But for higher dimension, the following theorem is not on the literature.

Theorem 12. Let $M=H^{n} / \Gamma$ be a convex cocompact hyperbolic $n \geq 3$ manifold. If $N$ is a convex cocompact hyperbolic $n$-manifold homotopy equivalent to $M$ so that corresponding ends are isometric by isometries preserving the markings, then it is isometric to $M$.

Proof: Let $\phi: M \rightarrow N$ be a proper homotopy equivalence extending an isometry on ends. Then its lift $\tilde{\phi}: H^{n} \rightarrow H^{n}$ extends to $S_{\infty}^{n-1}$ as a quasi-conformal map $\phi^{\prime}: S_{\infty}^{n-1} \rightarrow S_{\infty}^{n-1}$. Then $\phi^{\prime}$ is differentiable almost everywhere. The pullback of the spherical metric $\sigma$ by $\phi^{\prime}$ determines an ellipsoid in the tangent space to almost every point of $S_{\infty}^{n-1}$. The 
vectors maximizing the ratio $\left(\phi^{\prime}\right)^{*} \sigma(v) / \sigma(v)$ span a canonical subspace $E_{x} \subset T_{x} S_{\infty}^{n-1}$, which cuts the ellipsoid in a round sphere of maximum radius. If $\phi^{\prime}$ is not conformal there is a positive set on which $E_{x}$ has constant rank, which defines a $k$-plane field invariant under $\Gamma$.

By Lemma 5 and Sullivan's theorem, $\Gamma$ cannot have an invariant $k$ plane on the limit set. Now it suffices to show that $\phi^{\prime}$ is conformal on the domain of discontinuity. But it follows from the assumption that the corresponding ends are isometric.

The other application is the following. Note here that we are using a smooth convex boundary, not the boundary of the convex core.

Theorem 13. Let $M$ be a n-manifold, $n>3$, which admits a convex cocompact hyperbolic metric of constant sectional curvature. Suppose $M$ admits a hyperbolic metric with a convex smooth boundary. Then the induced metric on the boundary determines the hyperbolic metric uniquely.

Proof: First we will show that the induced metric determines the second fundamental form. But it is well-known that a hypersurface in $H^{n}$ is completely determined by its induced metric and the second fundamental form. In conclusion, a hypersurface in $H^{n}, n>3$ is completely determined by its induced metric. Once this is proved, we can prove the theorem as follows. Lift $\mathrm{M}$ with a convex smooth boundary into $H^{n}$. Then it is a domain $U$ bounded by hypersurfaces corresponding to boundary components of $M$ in $H^{n}$. Since each hypersurface $H$ is completely determined by its induced metric, a component of $H^{n} \backslash U$ bounded by $H$ is completely determined. Its quotient under the stabilizer of $H$ descends to an end of $M$. So each end of $M$ is completely determined by the induced metric on the boundary. Then by Theorem 12, the hyperbolic metric on $M$ is uniquely determined.

Hence it suffices to show that the induced metric determines the second fundamental form for dimension $>3$.

Let $I I$ be the real-valued second fundamental form and $B$ symmetric operators defined by

$$
I I(x, y)=I(B x, y)=I(x, B y) .
$$

Or if $D$ is the Levi-Civita connection on $H^{n}$ and $S$ is a hypersurface in it, then the shape operator $B: T S \rightarrow T S$ is defined by $B x=-D_{x} N$ where $N$ is a unit normal vector field to $S$ and it satisfies $I I(x, y)=$ $I(B x, y)=I(x, B y)$.

One can diagonalise $B$ with respect to an orthonormal basis $e_{i}$ with eigenvalues $b_{i}$. But the eigenvalues of $B$ can be deduced from the 
sectional curvatures of the induced metric. By the Gauss formula

$$
K(x, y)=\tilde{K}(x, y)+\bar{K}(x, y)
$$

where $\tilde{K}$ is the sectional curvature of $H^{n}$, which is -1 in this case, and

$\bar{K}$ is the Gaussian curvature. If $\left\{e_{i}\right\}_{i=1}^{n-1}$ is an orthonormal basis for the hypersurface which diagonalize $I I$, we have

$$
K\left(e_{i}, e_{j}\right)=-1+b_{i} b_{j} .
$$

If $n-1 \geq 3, b_{i}$ has a solution.

Hence for a given induced metric, one can diagonalise the second fundamental form using an orthogonal basis, and the eigenvalues can be calculated from the sectional curvatures, so the second funamental form is determined by the induced metric.

\section{REFERENCES}

[1] I. Agol, Tameness of hyperbolic 3-manifolds, preprint.

[2] R. Benedetti and C. Petronio, Lectures on Hyperbolic Geometry, Universitext, Springer, 1992.

[3] G. Besson, G. Courtois, S. Gallot, Entropies et rigidités des espaces localement symétriques de courbure strictement négative, GAFA. 5 (5) (1995), 731-799.

[4] F. Bonahon, Bouts des variétés de dimension 3, Ann. of Math. (2), 124 (1) (1986), 71-158.

[5] M. Bridgeman and R. D. Canary, From the boundary of the convex core to the conformal boundary. Geom. Dedicata 96 (2003), 211-240.

[6] M. Burger and R. Canary, A lower bound on $\lambda_{0}$ for geometrically finite hyperbolic $n$-manifolds, J. Reine angew. Math. 454 (1994), 37-57.

[7] R. Brooks, P. Perry and P. Petersen, Spectral geometry in dimension 3, Acta Math., vol 173 (2) (1994), 283-305.

[8] P. Buser, A note on the isoperimetric constant, Ann. Sci. École. Norm. Sup. 15 (1982), 213-230.

[9] P. Buser and G. Courtois, Finite parts of the spectrum of a Riemann surface, Math. Ann. vol 287 (3) (1990), 523-530.

[10] D. Calegari and D. Gabai, Shrinkwrapping and the taming of hyperbolic 3manifolds, J. AMS, 19 (2006), no 2, 385-446.

[11] R.D. Canary, Hyperbolic structures on 3-manifolds with compressible boundary, Ph.D thesis, Princeton University, 1989.

[12] R.D. Canary, On the Laplacian and the geometry of hyperbolic 3-manifolds, J. Differential geometry, 36 (1992), 349-367.

[13] R.D. Canary, Ends of hyperbolic 3-manifolds, J.AMS, 6 (1993), 1-35.

[14] R.D. Canary, A covering theorem for hyperbolic 3-manifolds and its application, Topology, 35 (1996), 751-778.

[15] R.D. Canary, Y.N. Minsky and E.C. Taylor, Spectral theory, Hausdorff dimension and the topology of Hyperbolic 3-manifolds, Jour. of Geometric Analysis, vol. 9 (1999), no. 1, 17-39.

[16] R.D. Canary and E. Taylor, Kleinian groups with small limit sets, Duke Math. J. vol 73 (1994), 371-381. 
[17] C. Chabauty, Limite d'ensemble et géométrie des nombres, Bull. Soc. Math. France, vol 78 (1950), 143-151.

[18] K. Corlette, Flat G-bundles with canonical metrics, J. Diff. Geom. 28 (1998), no. 3, 361-382.

[19] C. Croke, Rigidity for surfaces of non-positive curvature, Comment. Math. Helv. 65 (1990), 150-169.

[20] C. Croke and V. Sharafutdinov, Specral rigidity of a compact negatively curved manifold, Topology, vol 37 (6) (1998), 1265-1273.

[21] D. DeTurck and C. Gordon, Isospectral deformations II. Trace formulas, metrics and potentials, Comm. Pure Appl. Math. 42 (1989), 1067-1095.

[22] C. Gordon and E. Wilson, Isospectral deformations of compact solv-manifolds, J. Diff. Geom, 19 (1984), 241-256.

[23] M. Gromov, Metric structures for Riemannian and non-Riemannian spaces, Progress in Math, 152. Birkhäuser, Boston, MA, 1999.

[24] D. Herron, X. Liu and D. Minda, Ring domains with separating circles or separating annuli, J. Analyse Math., 53 (1989), 233-252.

[25] J. Jost, Riemannian Geometry and Geometric Analysis, Third edition. Universitext. Springer-Verlag, Berlin, 2002.

[26] Inkang Kim, Coordinates of the representation space in the semisimple Lie group of ran one, Bull. Austral. Math. Soc, vol 58 (3) (1998), 435-444.

[27] Inkang Kim, Geometry on exotic hyperbolic spaces, J. Korean Math. Soc, 36 (1999), no. 3, 621-631.

[28] Inkang Kim, Marked length rigidity of rank one symmetric spaces and their product, Topology, vol 40 (6) (2001), 1295-1323.

[29] Inkang Kim, Isospectral finiteness on hyperbolic 3-manifolds, Comm. Pure. Appl. Math., vol 59 (5) (2006), 617-625.

[30] F. Labourie, Cross ratios, Anosov representations and the energy functional on Teichmüller space, Annales Scientifiques de l'ENS, IV (2008).

[31] K. Matsuzaki and M. Taniguchi, Hyperbolic manifolds and Kleinian Groups, Oxford, 1998.

[32] D. McCullough, Twist groups of compact 3-manifolds, Topology, vol 24 (4) (1985), 461-474.

[33] D. McCullough and A. Miller, Homeomorphisms of 3-manifolds with compressible boundary, Memoirs of the A.M.S. 344 (1986).

[34] H.P. McKean, Selberg's trace formula as applied to a compact Riemann surface, Comm. Pure. Appl. Math., 25 (1972), 225-246.

[35] C.T. McMullen, Renormalization and 3-Manifolds which fiber over the circle, Annals of Math. Studies 142. Princeton, 1996.

[36] J. Morgan, On Thurston's uniformization theorem for three-dimensional manifolds. The Smith conjecture (New York, 1979), 37-125. Pure and Applied Mathematics, 112. Academic Press, Orlando, 1984.

[37] B. Osgood, R. Phillips and P. Sarnak, Compact isospectral sets of surfaces, J. Funct. Anal, vol 80 (1) (1988), 212-234.

[38] J.-P. Otal, Le spectre marqué des surfaces à courbure négative, Ann. of Math. (2) 131 (1990), 151-162.

[39] R. Spatzier, On isospectral locally symmetric spaces and a theorem of Von Neumann, Duke Math. J., 59 (1989), 289-294. 
[40] D.B.A. Epstein and A. Marden, Convex hulls in hyperbolic space, a theorem of Sullivan, and measured pleated surfaces, Analytical and geometric aspects of hyperbolic space ( Coventry/Durham, 1984), 113-253, LMS Lecture Note Ser., 111, Cambridge Univ. Press, Cambridge, 1987.

[41] J. Sacks and K. Uhlenbeck, Minimal immersions of closed Riemann surfaces, Trans. AMS, 271 (1982), 639-652.

[42] D. Sullivan, On the ergodic theory at infinity of an arbitrary discrete group of hyperbolic motions. In Riemann surfaces and Related Topics: Proceedings of the 1978 Stony Brook Conference. Annals of Math. Studies 97. Princeton, 1981.

[43] T. Sugawa, Various domain constants related to uniform perfectness, Complex Variables Theory Appl. 36 (1998), 311-345.

[44] T. Sunada, Riemannian covering and isospectral manifolds, Ann. Math. 121 (1985), 169-186.

[45] W. Thurston, The geometry and topology of 3-manifolds, Princeton Lecture notes.

[46] W. Thurston, Hyperbolic structures on 3-manifolds III: Deformations of 3manifolds with incompressible boundary, preprint.

[47] C.B. Yue, The ergodic theory of discrete isometry groups on manifolds of variable negative curvature, Trans. of AMS, vol 348 (12) (1996), 4965-5005.

[48] Y. Colin-de-Verdiére, Spectre du laplacien et longueurs des géodésiques périodiques I, II (French), Compositio Math. 27 (1973) 83-106; ibid. 27 (1973) 159-184.

[49] M.F. Vinéras, Variét'es riemanniennes isospectrales et non isométriques, Ann. Math. 112 (1980), 21-32.

\section{Gilles Courtois}

L'Institut de Mathmatiques de Jussieu

4, place Jussieu, 75252 Paris Cedex 09, France

e-mail: courtois@math.jussieu.fr

\section{Inkang Kim}

KIAS, School of Mathematics

Hoegiro 85, Dongdaemun-gu

Seoul, 130-722 Korea

e-mail: inkang@kias.re.kr 